\title{
Funktionelle Störungen der Gallenwege
}

\author{
Das Beschwerdebild bei Gallenwegsdyskinesien umfasst Übelkeit, \\ Völlegefühl und Beschwerden im rechten Oberbauch, die in den Rücken \\ oder bis zur rechten Schulter ausstrahlen und sich bis zu leichten Koliken \\ steigern können. Die Ätiologie ist bisher unklar, konventionelle Therapien \\ einschließlich der Cholezystektomie sind oft nicht erfolgreich.
}

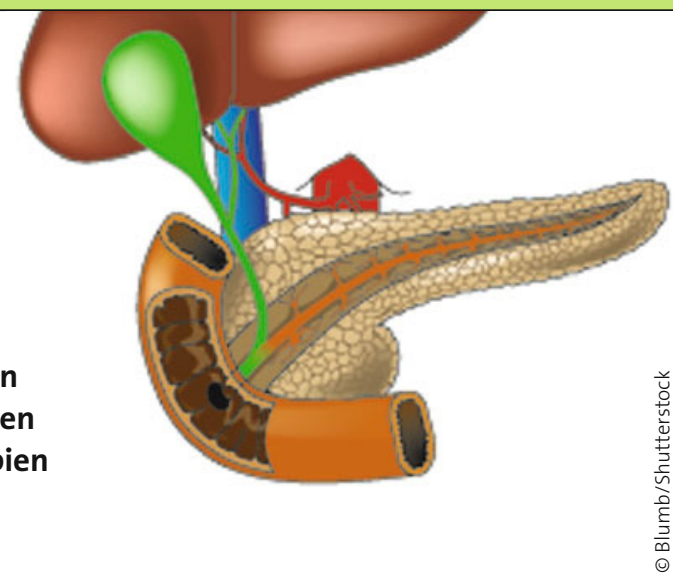

- Die Phytotherapie bietet sehr gute Therapieoptionen und ist zumeist als alleinige Behandlung ausreichend. Pflanzliche Cholagoga enthalten ätherische Öle, Bitter- und Scharfstoffe, die choleretisch und/oder cholezystokinetisch wirken. Viele dieser pflanzlichen Drogen wirken zudem antiphlogistisch, karminativ, antibakteriell und spasmolytisch.

Bewährt bei Motilitäts- und Sekretionsstörungen sind Artischockenblätter, Curcumawurzelstock, javanische Gelbwurz, Löwenzahnwurzel mit -kraut, Schafgarbenkraut und -blüten, schwarze Rettichwurzel, Pfefferminzöl/-blätter und Wermutkraut. Aus Rettich lässt sich Presssaft herstellen: den Rettich schälen, zerkleinern und auspressen, kühlen, vor Gebrauch etwas Zucker oder Leinsamenschleim hinzufügen; tgl. 100-150 $\mathrm{ml}$ Saft in kleinen Portionen einnehmen, nach vier bis fünf Tagen zwei bis drei Tage pausieren. Für Wermutkrauttee übergießt man $1 \mathrm{TL}$ mit $150 \mathrm{ml}$ heißem Wasser und lässt 5 min ziehen, abseihen; dreimal tgl. eine Tasse trinken. Um eine gute Wirksamkeit zu erhalten, sollte der

\section{Diätempfehlung}

Keine raffinierten Fette

Ernährungstherapie sollte mit Phytotherapie kombiniert werden. Insbesondere sollten leichtverdauliche Fette verwendet werden, d.h. nicht raffinierte Fette mit niedrigem Schmelzpunkt wie Butter, Sahne und kalt gepresste Öle.
Tee nicht gesüßt werden. Wermutkraut sollte nicht länger als drei bis vier Wochen angewendet werden.

\section{Kombinierte Phytotherapie}

Die Verwendung von Kombinationen ist sinnvoll, da es sich meist um ein komplexes Geschehen handelt. Die im Handel befindlichen Tee(-mischungen) und Tinkturen sollten bevorzugt werden. Pfefferminzöl sollte in Form von magensaftresistenten Arzneiformen angewendet werden (Einzeldosis $>100 \mathrm{mg}$ ).

Bei Übelkeit, Völlegefühl, Schmerzen im rechten Oberbauch und ungenügender Fettverdauung werden choleretisch und cholezystokinetisch wirksame Monopräparate oder Kombinationspräparate aus Artischockenblättern, Curcumawurzelstock, javanischer Gelbwurz, Harongarinde/-blättern, Kamillenblüten, Kümmelöl, Löwenzahnwurzel mit -kraut, Pfefferminzöl/-blättern und Wermutkraut verwendet. Artischockenblätter wirken zudem cholesterinsenkend, spasmolytisch, antiphlogistisch, Curcumawurzelstock spasmolytisch und antiphlogistisch, Harongarinde/-blätter sekretionsfördernd auf Magen- und Pankreas, javanische Gelbwurz antiphlogistisch und antibakteriell.

\section{Kontraindikationen und Nebenwirkungen}

Bei Cholelithiasis, Cholangitis oder intrahepatischer Cholestase, Verschluss der Gallenwege und Ikterus sind Cho- lagoga kontraindiziert. Artischockenblätter, Curcumawurzelstock, und Löwenzahnwurzel und -blätter sind kontraindiziert bei Lebererkrankungen und gleichzeitiger Einnahme leberbelastender Medikamente. Schafgarbenkraut und -blüten sind bei Überempfindlichkeit gegen Korbblütler zu meiden.

An unerwünschten Wirkungen können bei Pfefferminzöl Magenbeschwerden auftreten, bei Rettich und Löwenzahnwurzel Sodbrennen und Aufstoßen. Kombinationen mit Anthranoiddrogen können krampfartige Magen-Darm-Beschwerden verursachen, deshalb nicht zu Beginn der Therapie verwenden.

\section{Physikalische Therapie}

Hauptziele der physikalischen Therapie sind Spasmolyse (Schmerzlinderung), Entzündungshemmung und verbesserte Leberperfusion. Die Hydro- und Thermotherapie nutzt kutiviszerale Reflexe: Die mit einem feuchten Tuch umwickelte heiße Wärmflasche oder der Heublumensack lindern kolikartige Beschwerden. Spastische Beschwerden sprechen auch gut auf eine Bindegewebsmassage oder Periostbehandlung (Serie mit sechs Therapien) an. Sehr zu empfehlen ist die Kolonbehandlung, die ggf. vom Patienten selbst erlernt werden kann. Das Trinken von Sulfatwässern (im Rahmen von Kuraufenthalten) wirkt supportiv.

- Prof. Dr. med. Karin Kraft, Lehrstuhl für Naturheilkunde, Universität Rostock 